\title{
Research on Intelligent Control of Electric Drive System
}

\author{
Ming $\mathrm{Hu}$ \\ Nanchang Institute of Science \& Technology, Jiangxi Nanchang 330108
}

\author{
Keywords: Electric drive; Intelligence control; Fuzzy control; Single neuron control
}

\begin{abstract}
With the development of modern control theory, the general techniques of controller design is gradually replaced by artificial intelligence software technique be widely used. The common feature of the traditional general techniques and the artificial intelligence techniques is that different number and classes knowledge used for describing system performance are needed in them. Comparatively speaking, artificial intelligence software technique have more easy operation and better performance. The intelligent control of electric drive system has attracted much attention at present. This article elaborate all the general problems in this research direction systematically. This article explain the significance and method of adopting intelligence control in electric drive system at first, and then introduce the characteristics of fuzzy control and neuron control applied in electric drive system as two kinds of general intelligence control methods and provide the relevant experimental results.
\end{abstract}

\section{Introduction}

Intelligence control is a new stage of the development of the automatic control science. At present, the research and application of intelligence control have developed in many fields. At the same time, its development bring new ideas and new methods for the control strategy of electric drive system.

Like other subjects, intelligence control is produced and developed from the practices for solving important engineering and technical problems because of the requirement of the development of science and technology. With the improvement of degree of automation and the population of automation, control object become increasingly complex. For the processes in which mathematical model is hard to get or be complex, a fairly good result can not be get by the classic and modern control theories, even there is absolutely nothing it can give for them. But the skilled operation staff can operate freely in manual control. However, human generate the idea naturally that the experience of the skilled staff can be used in automatic control technology as reference. The development of computer control technology make the desire to be true. Computer can take the control work according to the experience and methods of the skilled staff and experts based on its functions in logical reasoning, judgment, recognition, decision-making, study and other aspects. On the other hand, a great deal of progress has been made in research of many academic fields such as artificial intelligence, experts system, nerve net and fuzzy logic in which how to carry out human brains function is explored, various control methods imitating people's knowledge and thought are proposed from different angles in these research productions such as expert controller, neuron control and fuzzy control which are collectively called intelligence control.

\section{Advantages of Artificial Intelligent Control System}

Artificial intelligent control system should adopt different control method according to different control. Nerve control, fuzzy control, neural network control and genetic algorithm can be seen as a family of nonlinear function approximator. These approximators can provide more accurate results and have more advantages than the conventional function estimator.

The specific model of control object will be needed in the control with the traditional function estimator, this is a big limitation, because it is very hard to get the exact dynamical equation of state in many occasions for human. But the limitation can not affect the artificial intelligent control system which do not need the model of control object.

Appropriate adjustment can improve the performance of artificial intelligent control system. For 
example, the rise time of fuzzy logic controller is 1.5 times as fast as optimizer, the fall time of fuzzy logic controller is 3.5 times as fast as optimizer, and fuzzy logic controller has smaller minimum voltage.

Artificial intelligent controller can be divided into three classes supervised, unsupervised and reinforcement learning controller. The topology structure and learning algorithm of traditional supervised learning neural network controller have become fixed and unchangeable, this point must give more limitations to controller with this structure that the computation time is more long and the computation effect is not good well. Applying neural network and cut-and-try method can avoid these errors in order to increase learning rate.

\section{Fuzzy Control}

Fuzzy control is a control method based on the fundamental principle of fuzzy mathematics. In traditional control technology fields, the precision of dynamic mode of control system is the key point affect the effect of control, more specific dynamic information, higher precision. However, for complex system, the dynamic process of system can not be described exactly because of too many variables included. Engineers try their best to reduce dynamic process of technology for precision control, but the results are not very satisfactory.

That is to say, the tradition control system theories have strong control for simple system, but have no strong control for complex system. Just for this reason, fuzzy mathematics was proposed by scientists in order to control the creation of these problems. Fig. 1 show the frame structure of general control system which contains five parts defined variable, fuzzification, knowledge base, logic judgment and defuzzification. 


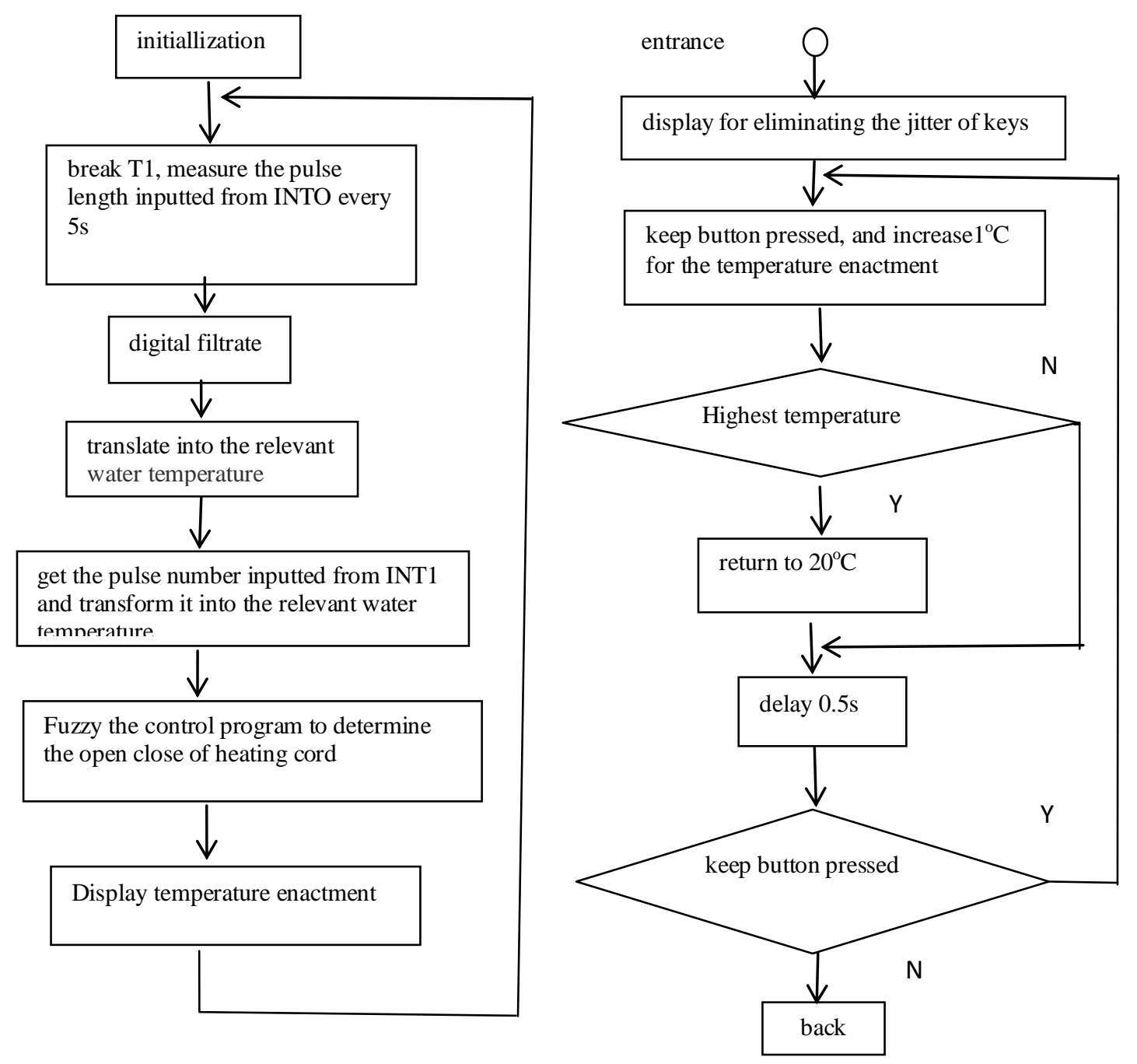

Figure 1. the frame structure of general control system

Defined variable: Defined variable is determination for the situations when program is observed and the actions of consider control. For example, for general problems of control, output error and the variety rate of output error are input variable, but controlled variable is the input of the next state.

Fuzzification: Fuzzification is a process in which input value is transformed into the numerical value of universe in the proper proportions and the measurement of physical quantity is described by colloquial variable.

Knowledge base: Knowledge base includes two parts data base and rule bank. Data base provide the relevant definitions for processing fuzzy data, and rule bank is used for describing control object and strategy by some language control rules.

Logic judgment: Logic judgment is imitating the fuzzy concept when human make judgment and making fuzzy reasoning in order to get control signal for control. This part also is central to the complete fuzzy controller.

Defuzzification: Defuzzification is transforming the fuzzy values into the exact control signal for the input of system. 


\section{Electric Drive System Controlled by Single Neuron Control}

Theoretically nerve net have strong ability to synthesise information which can solve all the complex control problems if the calculating speed is fast enough. Unfortunately, there is no support of the corresponding neural network computer hardware. But electric drive system controlled by single neuron control have met the requirements for nonlinear control and strong robustness aim to the control features of electric drive system.

Single neuron is the basic control part in neural network controller. Its model is as shown in Fig.

2. In the figure, $\mathrm{T} 1$ is the input of controller, and $\omega_{1}(\mathrm{i}=1,2,3)$ is the corresponding weight, $\mathrm{Ku}$ is proportionality factor, $\mathrm{f}(\cdot)$ is $\mathrm{S}$ type excitation function with the maximum limit amplitude.

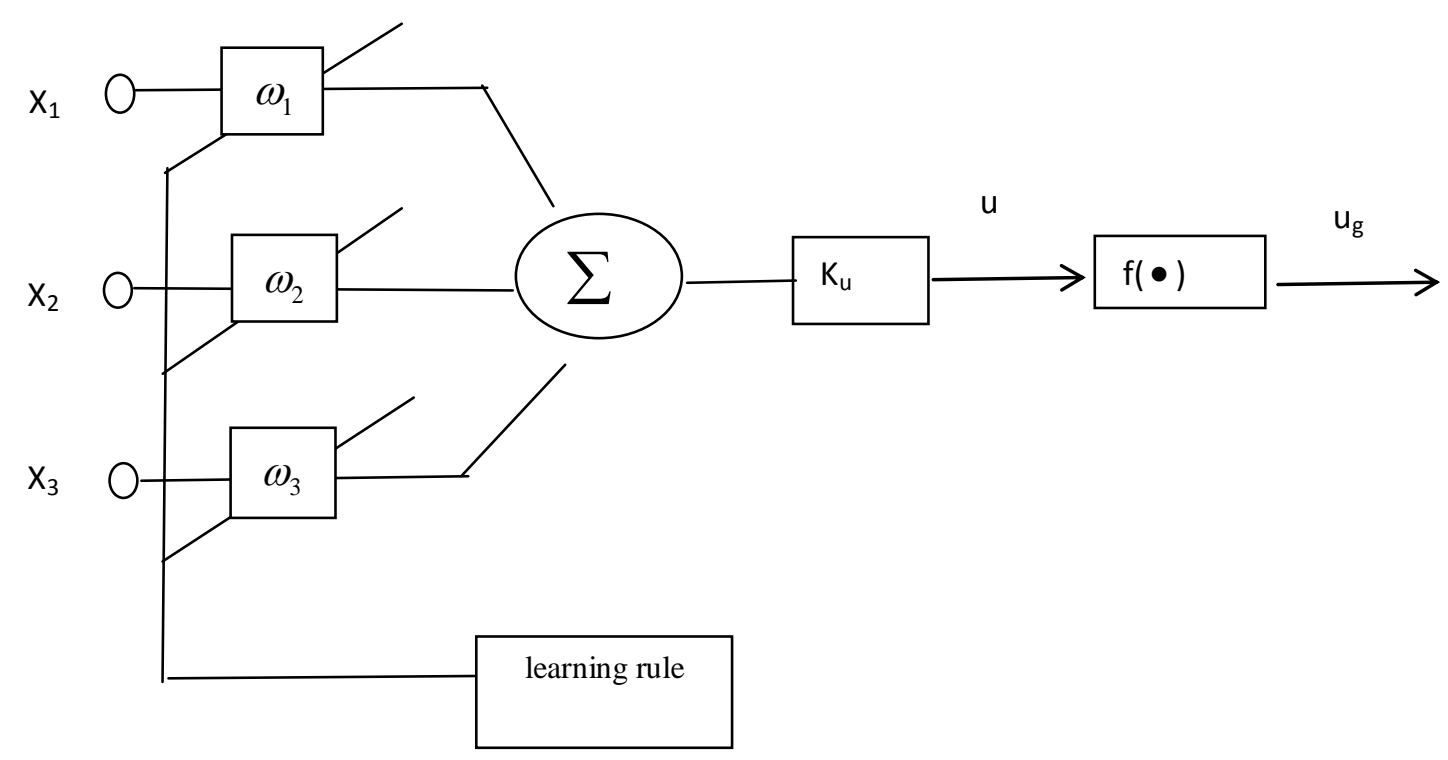

Figure 2. single neural model

in which,

$$
\begin{aligned}
& u(k)=K_{u} \sum_{i=1}^{3} \omega_{1}(k) x_{1}(k) /\|W\| \\
& u_{k}(k)=U_{\max } \frac{1-e^{-u(k)}}{1+e^{-u(k)}}
\end{aligned}
$$

In which, the inner product of weight vector $\mathrm{w}$ and input vector $\mathrm{x}$ is divided by the euclidean norm $\|\mathrm{W}\|$ of weight vector for the convergence of control strategy.

The self-adaptability function of single neuron controller is implemented by changing weight. Learning rule is adjusting the algorithm of weight w1 which is the core of single neuron controller, the algorithm is as below.

$$
\omega_{1}(k+1)=\varpi_{1}(k)+\eta_{1} v_{1}(k)
$$

in which,

$\eta_{1}>0$-learning rate

$v_{1}(k)$-learning signal decreasing with process

For drive system, considering the two running conditions of motor the forward and the reversal, the following learning methods are adopted to guarantee the convergence of learning algorithm. 


$$
v_{1}(k)=\left.\ell(k)\right|_{x}(k) \mid x_{1}(k)
$$

Suppose $\mathrm{X} 1$ is error, $\mathrm{X} 2$ is error integral, $\mathrm{X} 3$ is error differential, of course, they are all input.

Electric drive system with high performance and strong robustness can be implemented by automatically adjusting the weight of all input with the learning rule of neuron network to make the dynamic of system only depend on the error signal and with no effect or less effect of objective model parameters, single neuron is adaptive variable coefficient PID controller in this process. In addition, the controller use the nonlinear characteristic peculiar to neuron, break through limitation of linear adjuster and implement steady, saturate and control function of rotary speed controller.

\section{Conclusion}

Designing a intelligent controller or a traditional controller designed for a control object is nothing but creating a reflection relationship from the current state, error of system to the controlled quantity. The difference of intelligent control and traditional control is that the latter design controller according to model of controlled object, and intelligent control does not need model of controlled object. Intelligent control imitate human intelligence, determine the output of controller just according to system error and its changes and automatically adjust controller. The mathematical model of model drive system is clear basically just be affected by parameter changes and nonlinear factor, but intelligent control method which have adaptive variable parameter function can be applied to make up for the shortcomings of linear IPD adjuster, and to improve the adaption ability of system for various disturbance factors and nonlinear factors. In the current theoretical level, electric drive intelligence control system can be seen as a family of nonlinear system for using the existing nonlinear theory and tool.

\section{Acknowledgements}

Project funding: the Science and Technology Key Project of the Educational Department in Jiangxi Province (number: GJJ151234).

\section{References}

[1] Kim M, Metzner J J, A Key Exchange Method for Intelligent Electronic Devices in Distribution Automation[J]. IEEE Transactions on Power Delivery, 2010, 25(3):1458-1464.

[2] Ha B N, Lee S W and Shin C H, et al. Development of intelligent distribution automation system[C]// Transmission \& Distribution Conference \& Exposition: Asia and Pacific. IEEE, 2009:1-4.

[3] Q Sun, X Dong, Discussion on intelligent distribution automation system construction mode[J]. 2012, 8562(5):1-6.

[4] W Shi, S Feng and Y Xia, New intelligent power distribution automation terminal self-describing[J]. Automation of Electric Power Systems, 2012.

[5] H Shu, X.B Liang and X.H Gao, Key technologies and test method of Remote Terminal in intelligent distribution automation system[J]. 2010:1-6.

[6] Q Sun, X Dong, Discussion on intelligent distribution automation system construction mode[C]// China International Conference on Electricity Distribution. IEEE, 2012:1-6.

[7] S Huang, X.B Liang and X.H Gao, Key technologies and test method of Remote Terminal in intelligent distribution automation system $[\mathrm{C}] / /$ China International Conference on Electricity Distribution. IEEE, 2010:1-6. 
[8] Rudd S, Kirkwood J and Davidson E, et al. Intelligent monitoring of distribution automation[J]. Pac World, 2012.

[9] Rudd S E, Kirkwood J D and Davidson E M, et al. Intelligent monitoring of the health and performance of distribution automation[C]// Intelligent System Application to Power Systems (ISAP)， 2011 16th International Conference on. IEEE， 2011:1-6.

[10] S.X Feng, Discussion on distributed feeder automation technology in intelligent distribution network[J]. World Nonferrous Metals, 2016.

[11] S.X Feng, Discussion on distributed feeder automation technology in intelligent distribution network[J]. World Nonferrous Metals, 2016.

[12] S.X Feng, Discussion on distributed feeder automation technology in intelligent distribution network[J]. World Nonferrous Metals, 2016. 